\title{
A New Scaling Approach for the Mesoscale Simulation of Magnetic Domain Structures using Monte Carlo Simulations ${ }^{1}$
}

\author{
B. Radhakrishnan, M. Eisenbach and T. A. Burress
}

Oak Ridge National Laboratory, 1 Bethel Valley Road, Oak Ridge, Tennesse 37831, USA

Corresponding Author:

\author{
B. Radhakrishnan \\ e-mail address: radhakrishnb@ornl.gov \\ Postal Address: 1 Bethel Valley Rd, Oak Ridge, \\ Tennessee, 37831-6114
}

Key Words: Monte Carlo, Scaling, Domain, Simulation

\footnotetext{
${ }^{1}$ This manuscript has been authored by UT-Battelle, LLC under Contract No. DE-AC05000R22725 with the U.S. Department of Energy. The United States Government retains and the publisher, by accepting the article for publication, acknowledges that the United States Government retains a non-exclusive, paid-up, irrevocable, worldwide license to publish or reproduce the published form of this manuscript, or allow others to do so, for United States Government purposes. The Department of Energy will provide public access to these results of federally sponsored research in accordance with the DOE Public Access Plan (http://energy.gov/downloads/doepublic-access-plan).
} 


\section{Abstract}

A new scaling approach has been proposed for the spin exchange and the dipoledipole interaction energy as a function of the system size. The computed scaling laws are used in atomistic Monte Carlo simulations of magnetic moment evolution to predict the transition from single domain to a vortex structure as the system size increases. The width of a $180^{\circ}$ domain wall extracted from the simulated structures are in close agreement with experimentally values for an F-Si alloy. The transition size from a single domain to a vortex structure is also in close agreement with theoretically predicted and experimentally measured values for Fe.

\section{Background}

Soft magnetic materials are used in a variety of applications ranging from magnetic recording to electric motors and power transformers. These materials are characterized by a remnant magnetic structure that consists of magnetic domains regions in which all the atomic magnetic moments align in specific directions in space. The soft magnetic properties are determined by the ease with which the magnetic domains can respond to external magnetization and demagnetization magnetic hysteresis loop. Stable domain structures form due to the minimization of the excessively large demagnetization energy that develops when all the magnetic moments self align in a large volume of material and provide moments normal to free surfaces. Energy is minimized by having the moments break up into domains, and the presence of domain walls within which the moments gradually change orientation from one domain orientation to the other. The width over which the moments change direction or the domain wall width is a function of the exchange energy that tends to align the moments in one direction and the magneto-crystalline anisotropy energy that preferentially aligns the moments along the "easy" directions of magnetization in the material.

Simulation of magnetic domains in magnetic materials has largely involved numerical solution to the micro-magnetics equation proposed by Landau-Lifshitz and Gilbert (LLG) [1-5]. The LLG equation describes the temporal evolution of the magnetic moment in an effective magnetic field. The effective magnetic moment is obtained as the gradient of the total magnetic energy with respect to the magnetization vector. The total magnetic energy is represented as the sum of the spin exchange energy, $E_{e x}$, magneto-crystalline anisotropy energy, $E_{a n}$, energy due to external magnetic field, $E_{\text {ext }}$, and the dipole-dipole interaction energy, $E_{d i p}$. The minimum of the total energy is obtained by the time integration of the LLG equation. While the LLG approach is based on treating magnetism as a continuous vector field, simulations of nanoscale magnetic behavior have used discrete magnetic moments at the atomistic scale to predict their collective evolution using atomistic techniques [6]. Such an approach has been used for example in simulating magnetic domain formation in nanoscale thin films [7], and magnetic switching in nanoscale devices $[8,9]$. 
Of the above magnetic energy terms mentioned above, the one that is computationally expensive to simulate is $E_{d i p}$ due to its long-range effect. $E_{d i p}$ in the continuum vector field representation is given as [10]:

$$
E_{\text {dip }}=-\frac{1}{2} \int_{r} K_{d} \vec{h}_{d} d \vec{r}
$$

where, the energy coefficient $K_{d}$ is given by

$$
K_{d}=\frac{M^{2}(\vec{r}, t)}{\mu_{0}}
$$

and $\vec{h}_{d}$ is the dipole-dipole interaction among the magnetic moments given by

$$
\vec{h}_{d}(r)=-\int_{r^{\prime}} \frac{1}{4 \pi r^{\prime 3}}\left[3\left(\widehat{m}_{1} \cdot \overrightarrow{r^{\prime}}\right)\left(\widehat{m}_{2} \cdot \overrightarrow{r^{\prime}}\right)-\widehat{m}_{1} \cdot \widehat{m}_{2}\right] d \overrightarrow{r^{\prime}}
$$

where $\mathrm{M}$ is the magnitude of the moment vector, $\widehat{m}_{1}$ and $\widehat{m}_{2}$ are the unit vectors of the magnetic moments at positions 1 and 2 and $\overrightarrow{r^{\prime}}$ is the unit vector of the displacement between positions 1 and 2 and $\mu_{0}$ is the permeability of free space. Inspection of Eqns. 1 to 3 shows that the dipole-dipole interaction energy is an $\mathrm{O}\left(\mathrm{N}^{2}\right)$ calculation because at each point in the simulation volume, the calculations involves summation through all the points in the volume. There are two main strategies used to reduce the computational load: (1) using the convolution theorem in Fourier space and Fast Fourier Transforms (FFT) [1, 9-12], and (2) the use of Fast Multipole Method (FMM) to convert the $\mathrm{O}\left(\mathrm{N}^{2}\right)$ to $\mathrm{O}[\mathrm{NLog}(\mathrm{N})]$ calculations [13]. FFT techniques usually require the use of periodic boundary conditions, which may not be realistic for certain applications. However, the technique is highly suited for parallel computations with linear scaling using recently developed parallel libraries [14]. FMM method has been widely used in many O(N2) type calculations. However, its use in micro-magnetic simulations has been rather limited, largely because of the relatively larger effort in implementing it in a parallel computing environment. The main advantage of FMM over FFT methods is that there is no restriction on the boundary conditions that can be used.

An alternate simulation approach has been used to investigate magnetic behavior of nanoscale materials where the focus has been to predict the transition from a single domain to a vortex structure and the associated effects in nanoscale magnetic devices. This approach is based on extending atomistic Monte Carlo (MC) simulations to length scales of tens of nanometers where such a transition is normally observed. Due to the expensive computational cost associated with performing such large MC simulations, scaling approaches have been developed so that such transitions can be observed in smaller systems that can be predicted to occur in larger systems. In the past there have been two main scaling approaches. One such approach is based on reducing the exchange energy according to $E_{e x}^{\prime}=E_{e x}$ where $\mathrm{x}<1$, and performing the simulations in a smaller simulation domain, $\mathrm{L}^{\prime}$ 
which is related to the actual domain $\mathrm{L}^{2} \mathrm{~L}^{\prime}=\mathrm{Lx}^{\eta}$, where $\eta$ is a scaling parameter. A universal value for $\eta$ was shown to exist at 0.551 that was independent of $x$. The approach has been widely used to investigate nano-magnetic behavior in several applications [15]. However, the universality of $\eta$ has been questioned more recently, and a scaling approach based on the total energy rather than just $E_{e x}$ has been proposed [16]. The approach is based on scaling the total energy, and not just $E_{e x}$, according to $E_{\text {tot }}^{\prime}=\lambda E_{e x}^{\prime}+E_{d i p}^{\prime}$ and explore a scaling law for $\lambda$ according to $\lambda=x^{\beta}$ where $\beta$ is the scaling factor based on the total energy criterion. The simulations showed that the ratio $\beta / \eta$ was a constant equal to 1.8 and that a given system could be studied with different values of $\eta$, provided the above condition was satisfied. While the above scaling approaches are based on scaling down the exchange or the total energy, we present a new approach that is based on scaling up the various atomistic magnetic energy contributions. We demonstrate that for a specific geometry there is a reasonably unique scaling law for $E_{\text {dip }}$ as well as $E_{e x}$. By making use of the scaling laws, and by utilizing high performance computing (HPC) we are able to extend the simulation volume to micrometer length scales that allows us to capture the domain structures at these length scales.

\section{Computational Approach}

The MC technique uses atomic magnetic moments distributed in a body centered cubic (BCC) structure of iron. The four magnetic energy contributions are expressed in terms of the atomic quantities as shown below:

$$
\begin{aligned}
& E_{\text {ext }}=-H \cdot M \\
& E_{\text {exch }}=-\sum J_{i j} \widehat{m}_{i} \cdot \widehat{m}_{j} \\
& E_{\text {dip }}=M^{2} \sum_{j=1}^{n} \sum_{k \neq j}^{n} \frac{\hat{m}_{j} \cdot \hat{m}_{k}-3\left(\hat{m}_{j} \cdot e_{j k}\right)\left(\hat{m}_{k} \cdot e_{j k}\right)}{4 \pi \mu_{0} r^{3}} \\
& E_{\text {an }}=K_{1}\left(\alpha_{1}^{2} \alpha_{2}^{2}+\alpha_{2}^{2} \alpha_{3}^{2}+\alpha_{1}^{2} \alpha_{3}^{2}\right)+K_{2} \alpha_{1}^{2} \alpha_{2}^{2} \alpha_{3}^{2}
\end{aligned}
$$

In Eqn. $4, H$ is the external magnetic field strength and $M$ is the atomic magnetic moment vector. In Eqn. 5, $E_{\text {exch }}$ is for each atom $i$ where the $\widehat{m}_{i}$ and $\widehat{m}_{j}$ are the unit magnetic moment vectors of the $i$ and $j$ atoms where the $j$ atoms included are up to the fourth nearest-neighbors, and $J_{i j}$ is the exchange interaction parameter between $i$ and $j$ atoms. In Eqn. 6, $e_{j k}$ is the unit vector of the displacement that connects an atom $j$ with atom $k$, and $\mu_{0}$ is the permeability of space. In Eqn. 7, $K_{1}$ and $K_{2}$ are the magneto-crystalline anisotropy constants for iron, and $\alpha_{1}, \alpha_{2}$ and $\alpha_{3}$ are the direction cosines of the total atomic magnetic moment vector with respect to the coordinate axes.

In the current MC simulation approach, the total magnetic energy of the system is represented as the sum of the energy contributions arising from Eqns. 4 through 7. 
The Metropolis algorithm [17] was used for the acceptance probability, $p$, is defined as

$$
\begin{array}{cc}
p=1.0 & \Delta E \leq 0.0 \\
p=e^{\frac{-\Delta E}{k T}} & \Delta E>0.0
\end{array}
$$

where $\Delta E$ is the change in the energy due to an attempted MC move. The two MC moves considered are as follows: (1) a random rotation of the magnetic moment of a single atom and (2) a cluster move that involves the rotation of all atoms in the simulation domain by a specified angle. The MC simulations were carried out in a parallel computing environment using Message Passing Interface (MPI) for interprocess communications. Although parallel computing allows simulations to be carried out in large volumes, it would still be prohibitive to extend the atomic level simulations to micrometer length scale, especially because of the $0\left(\mathrm{~N}^{2}\right)$ nature of the $E_{\text {dip }}$ calculations. Therefore, an attempt was made to develop a scaling law for $E_{\text {dip. }}$. A similar approach was used to scale $E_{e x}$ so that the scaled values could be used in coarse- grained simulations. The scaling procedures are described in the following sections.

\subsection{Scaling of exchange energy}

In the atomic scale, the exchange interaction parameters for the first through the fourth nearest neighbor atoms are known from First Principles calculations [18]. The following expression obtained by fitting the results of the first-principles calculations was used in the current calculations:

$$
J\left(r_{n}\right)=0.5190575-0.367938 r_{n}+0.086530 r_{n}^{2}-0.0067552 r_{n}^{3}
$$

where $J(n)$ is the interaction energy for the $\mathrm{n}^{\text {th }}$ nearest-neighbor atom in $\mathrm{eV}$ and $\mathrm{r}_{\mathrm{n}}$ is the distance of the nth nearest atom in Anstroms. In the current simulations, the exchange interaction was limited to the first through the third nearest neighbor atoms.

The approach used for scaling the exchange energy was as follows. Initially all the magnetic moments within the simulation volume were oriented in the $z$-direction of the $x y z$ coordinate system, and the initial exchange energy was calculated. Then an atom at a specific location was selected, its moment was rotated by a random angle, $\theta_{R}$, and the change in the exchange energy due to rotation of the single atom was calculated. Next, the moments of all atoms belonging to $n \times n \times n$ unit cells positioned around the reference atom were rotated by $\theta_{R}$, and the energy change was calculated. This is assumed to correspond to a size scale of $n$. The above procedure was repeated for $n$ up to 200. For a given $n$ the unit cells considered had their centers ranging from $-\frac{(n-1)}{2}$ to $\frac{(n-1)}{2}$ along each coordinate axis. The simulations 
were carried out in a 1024 x 1024 x 1024 domain with a BCC crystal structure. The simulations required 512 processes to complete in a reasonable computational time frame. Figure 1 shows a schematic of the scaling procedure.
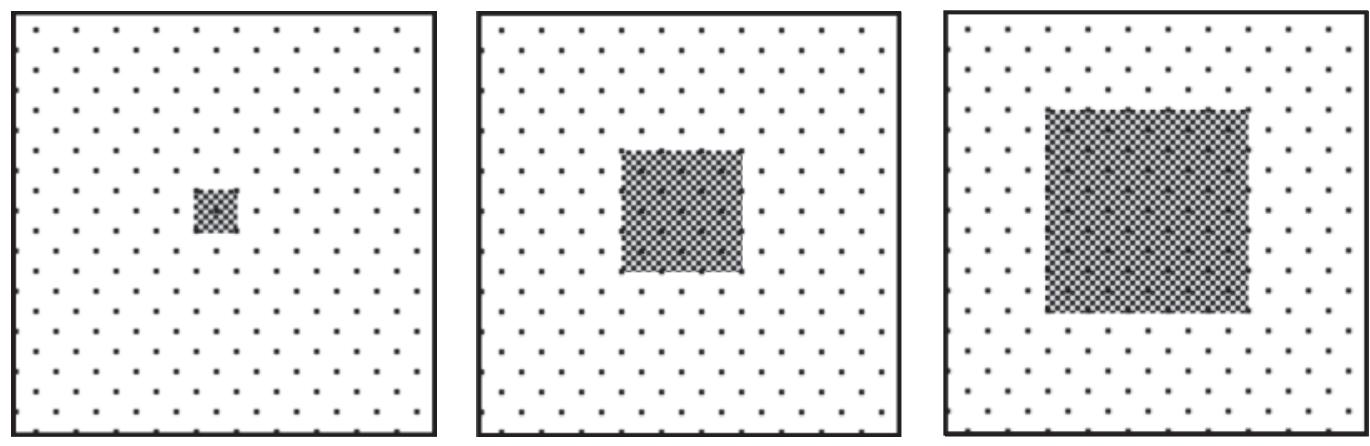

Figure 1. Schematic of the size scaling used for exchange and dipole-dipole interaction energies in 2-d. A similar scheme was in 3-d.

\subsection{Scaling of dipole-dipole interaction energy}

The simulation framework for the scaling of $E_{d i p}$ was the same as the one used for $E_{e x}$. However, $E_{\text {dip }}$ extends over much larger distances covering the entire computational domain. Therefore, in these simulations the simulation volume had to be increased proportionately with the size scale. For example, when $n=3$ the edges of the simulation domain were also increased by a factor of 3 in order to maintain constancy of the ratio of the simulation volume to the volume of atomic clusters of a scaled site. For each condition, $E_{\text {dip }}$ was calculated rigorously using $\mathrm{O}\left(\mathrm{N}^{2}\right)$ calculations. Such calculations required the use of parallel computing using up to 1024 processes.

\section{Results and Discussion}

\subsection{Scaling studies}

The objective of the scaling studies was to calculate the effective increase in $E_{e x}$ and $E_{d i p}$, when the atomic simulations are scaled such that each site in the coarsegrained simulation volume would correspond to a collection of unit cells of BCC as described above. If a unique scaling factor exists, then it is possible to extend the simulation length scale performed using atomistic length scale to much larger "effective" length scales. The variables that were considered in order to establish the uniqueness of the scaling curve included the rotation angle, $\theta_{R}$, and the position of the reference atom within the simulation volume. Figure 2 shows the summary of the simulation runs for the $E_{e x}$. It is clear that the scaling is unique and does not depend on the above-mentioned variables. Equation 10 below is a polynomial fit to the scaling data shown in Fig. 2.

$$
E_{\text {ex }}=0.1362+2.871 n+2.863 n^{2}
$$




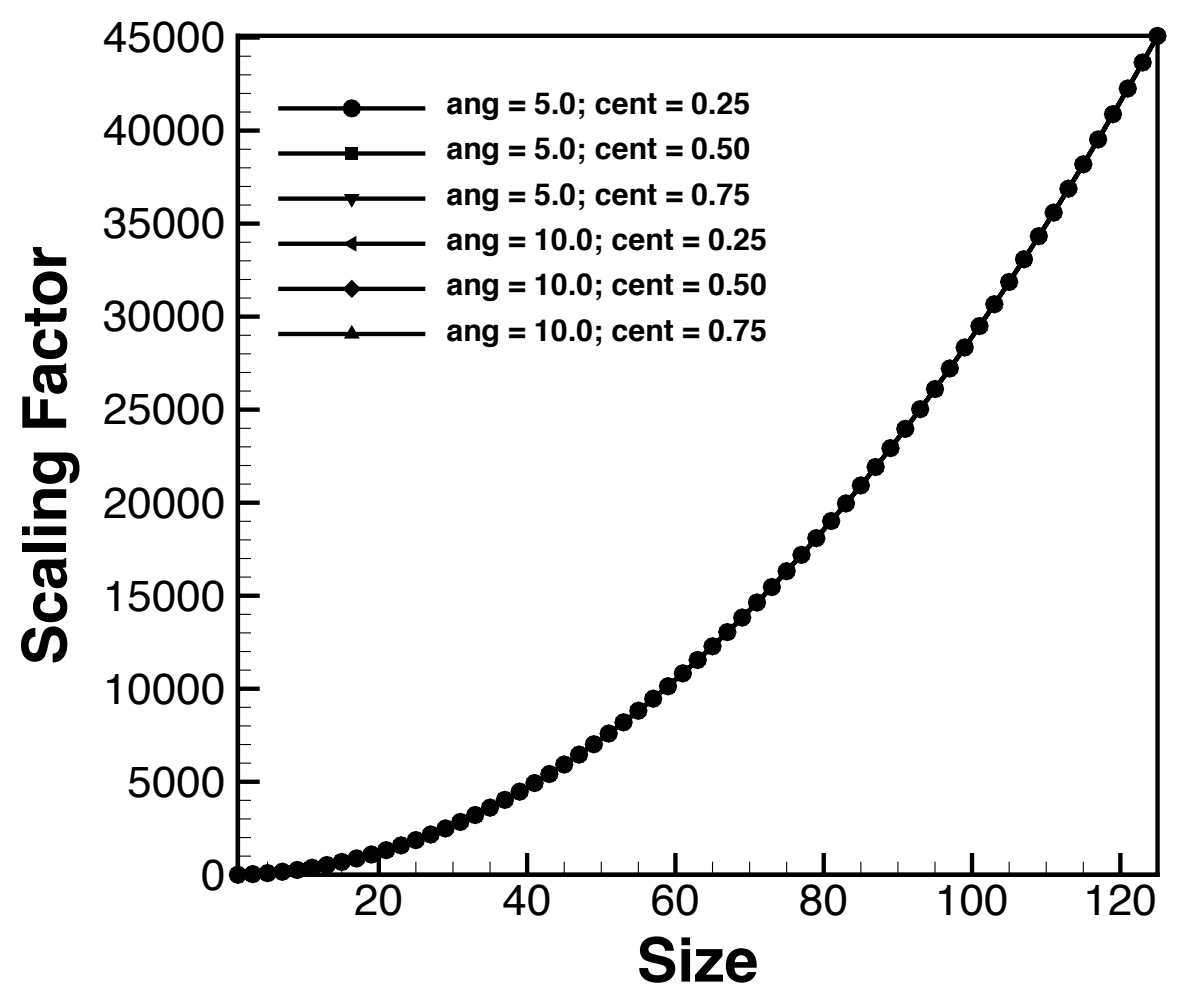

Figure 2. Scaling of the exchange interaction energy as a function of the size scale for different $\theta_{R}$ and positions of the reference atom.

Since $E_{e x}$ is localized, it was possible to investigate the scaling up to $n=200$ using a moderate parallel computing effort. However, $E_{\text {dip }}$ calculations using $\mathrm{O}\left(\mathrm{N}^{2}\right)$ approach became too expensive beyond a certain size scale even with the available computational resources.
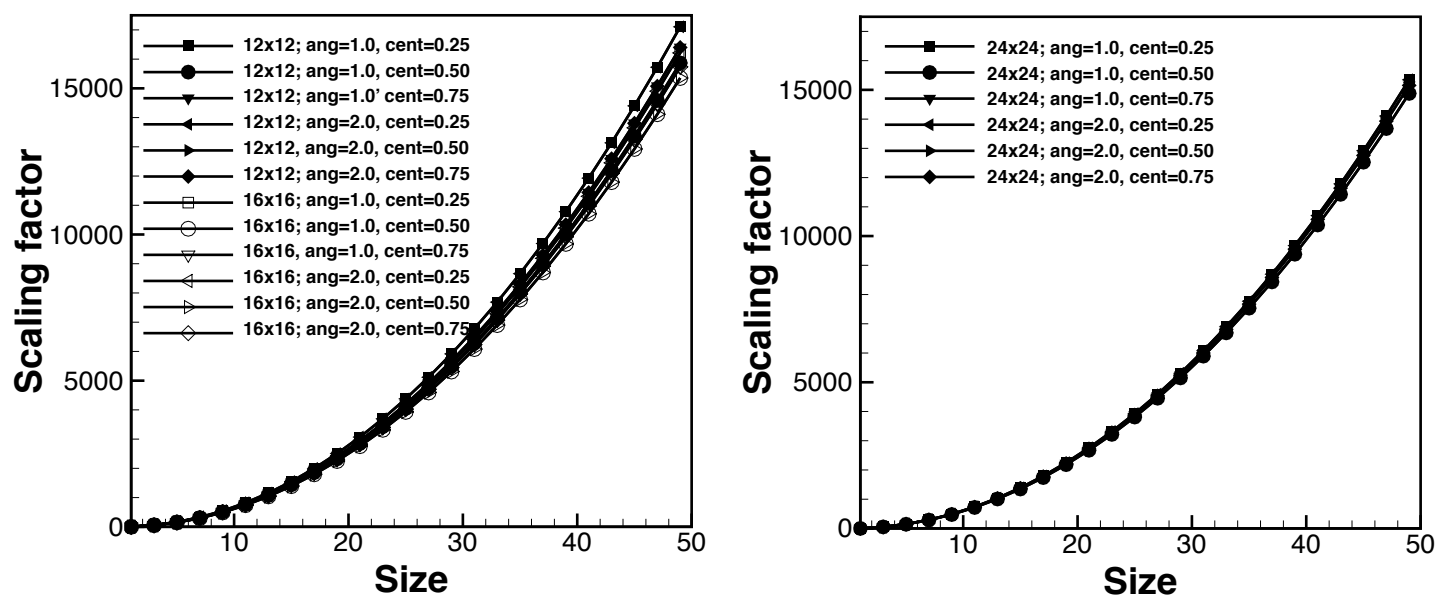
Figure 3. Scaling of the dipole-dipole interaction energy with size using 2-d simulations. Accuracy of the simulations improves with simulation size. Therefore, it was decided to first investigate the scaling of $E_{\text {dip }}$ using twodimensional $(2-d)$ simulations. Figure 3 is a summary of the $2-d$ simulation results. While there is some variation in the scaling curve with $\theta_{R}$ and relative position of the reference atom, the variation becomes much smaller as the simulation volume is increased. After establishing the existence of a unique scaling curve in 2- $d$ simulations, the scaling of $E_{d i p}$ was investigated in $3 \mathrm{~d}$ using relatively smaller computational domains because of the excessively high computational cost.

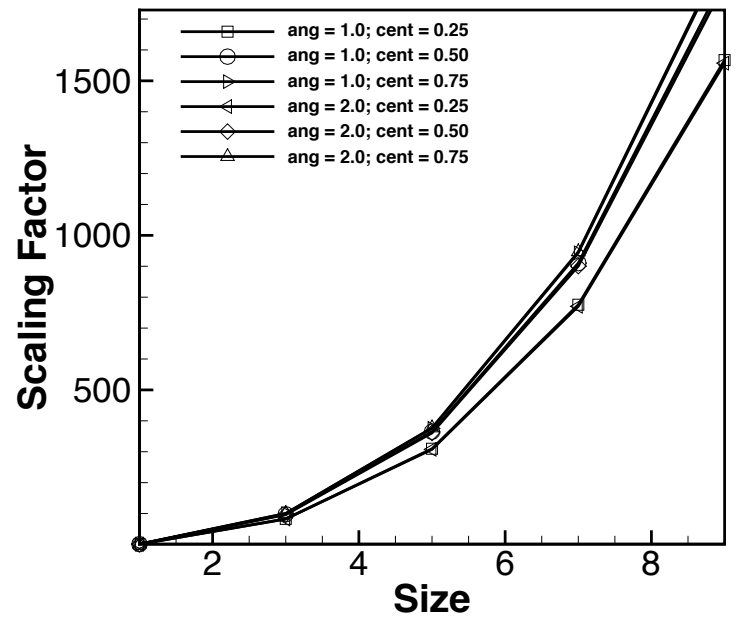

(a)

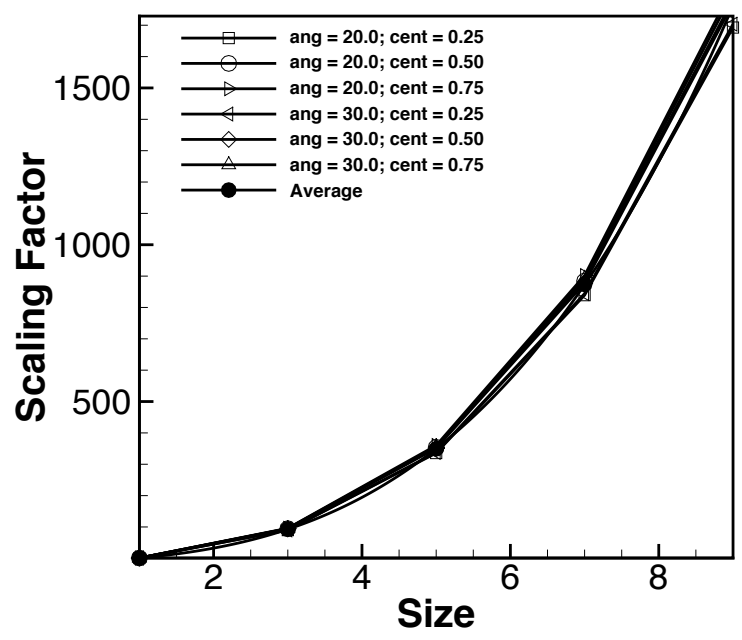

(b)

Figure 4. Scaling of the dipole-dipole interaction energy with size using 3-d simulations. Accuracy of the results improves with an order of magnitude increase in the rotation angle.

Figure 4 shows the results for $3 \mathrm{~d}$ simulations for 2 different ranges of the rotation angle. The results shown in Fig. 4a correspond to rotation angles $1^{\circ}$ and $2^{\circ}$ using a computational volume of $64 \times 64 \times 64$ atoms, which is a reasonable compromise between computational cost and volume. The scatter in the scaling curves as a function of $\theta_{R}$ and the position of the reference atom appears to be quite significant. However, when the range of the rotation angles is increased by an order of magnitude as shown in Fig. 4b, the scatter in the scaling is significantly reduced. The scatter in the scaling curves in Fig. 4 a appears to be related to the fact that $E_{d i p}$ is a much smaller quantity compared to $E_{e x}$ and the uncertainty associated with its calculation when $\theta_{R}$ is small. The uncertainty in scaling is reduced by using higher rotation angles shown in Fig. $4 \mathrm{~b}$ and the corresponding scaling curve is used in the subsequent coarse-grained simulations. The above simulations taken together seem to indicate that it is possible to scale $E_{e x}$ and $E_{\text {dip }}$ using a unique scaling curve independent of the location of the moment within the computational domain in the atomic level simulations. Equation 11 is a polynomial fit to the average curve shown in Fig.4b. 


$$
E_{\text {dip }}=-14.097+11.299 n+1.829 n^{2}+2.094 n^{3}
$$

From Eqns. 10 and 11, it is clear that the dipole-dipole interaction energy scales much more steeply with simulation size than the exchange interaction. This is the key for domain formation. Beyond a critical size, the high demagnetization energy due to the aligning of the moments normal to a free surface can be reduced by the gradual rotation of the moments to form a domain wall inside the simulation volume. Atomic level simulations along with the use of the scaling factors were used to demonstrate the formation of domain structures and compared with existing results in the literature obtained using phase field simulations.

\subsection{Transition from Single Domain to Vortex Structure}

An appropriate validation of the scaling studies is the simulation of the transition from a single domain to a multiple domain structure when the size of the magnetic particle is increased $[19,20]$. If the particle size is smaller than the domain wall width, then the particle tends to be a single domain. As the particle size is increased, there is a transition from a single domain structure to a vortex structure to a well defined multiple domain structure. The results of such a transition based on the application of the scaling laws for $E_{e x}$ and $E_{a n}$ are shown in Fig. 5. The simulations were carried out in a relatively small atomistic domain consisting of $32 \times 32 \times 32$ atoms situated in BCC structure. The scaling factor was varied by increasing the size (Figs. 2-4), and the effective $E_{e x}$ and $E_{d i p}$ were calculated based on the fitted polynomials shown in Eqns. 10 and 11, respectively. When the size $n=1.0$, each moment shown represents an atomic moment. The moments are all aligned along one of the easy magnetization directions, $<100>$ type directions (Fig. 5a).

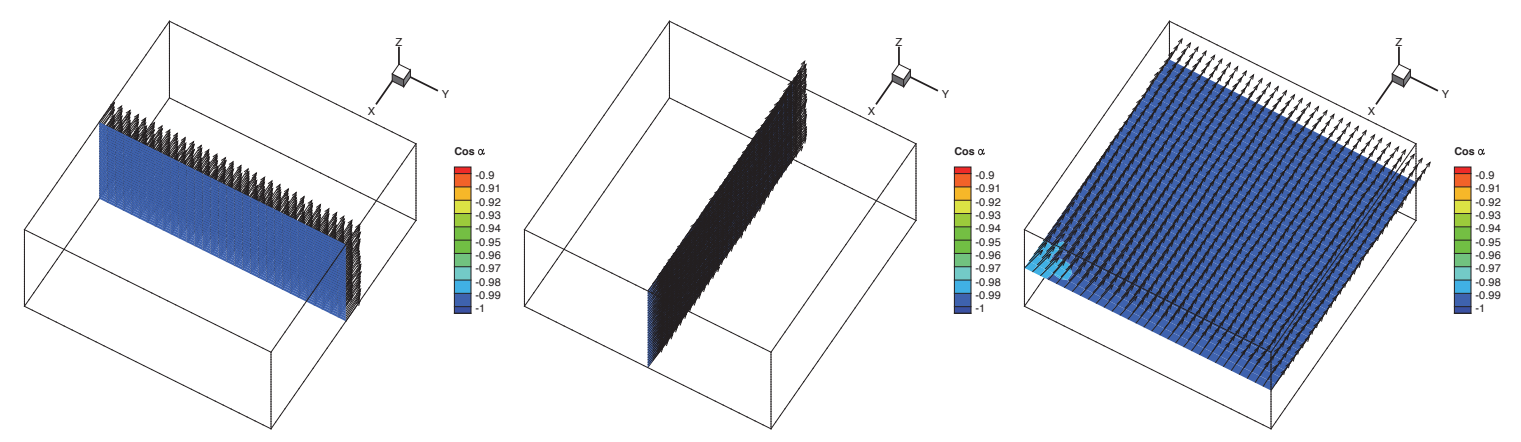

(a) Size $=1\left(9.152 \times 9.152 \times 4.576 \mathrm{~nm}^{3}\right)$ 


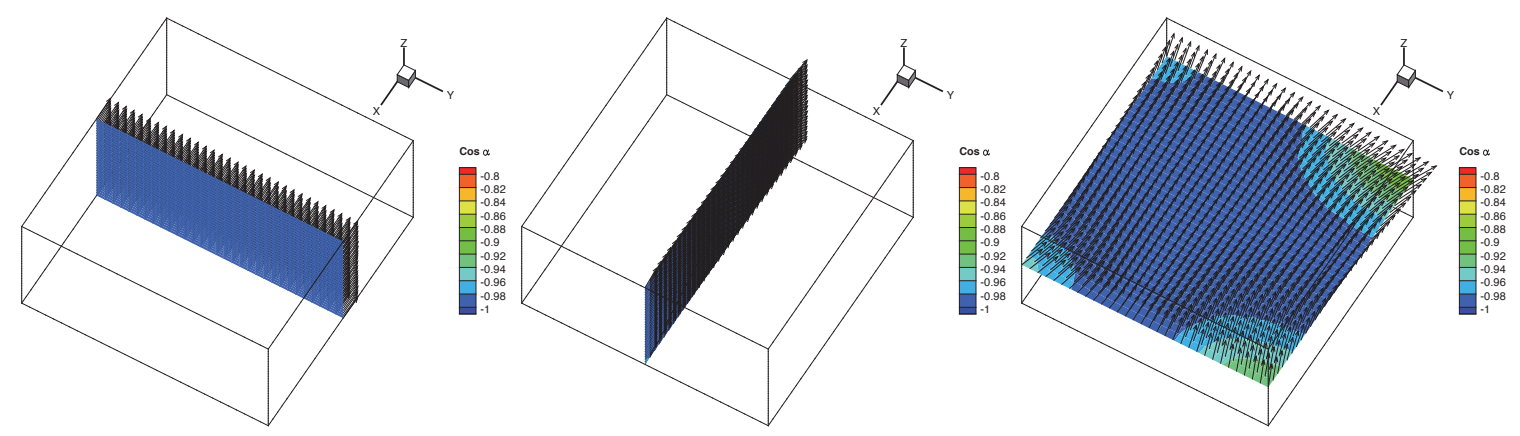

(b) Size $=11\left(100.6 \times 100.6 \times 50.3 \mathrm{~nm}^{3}\right)$

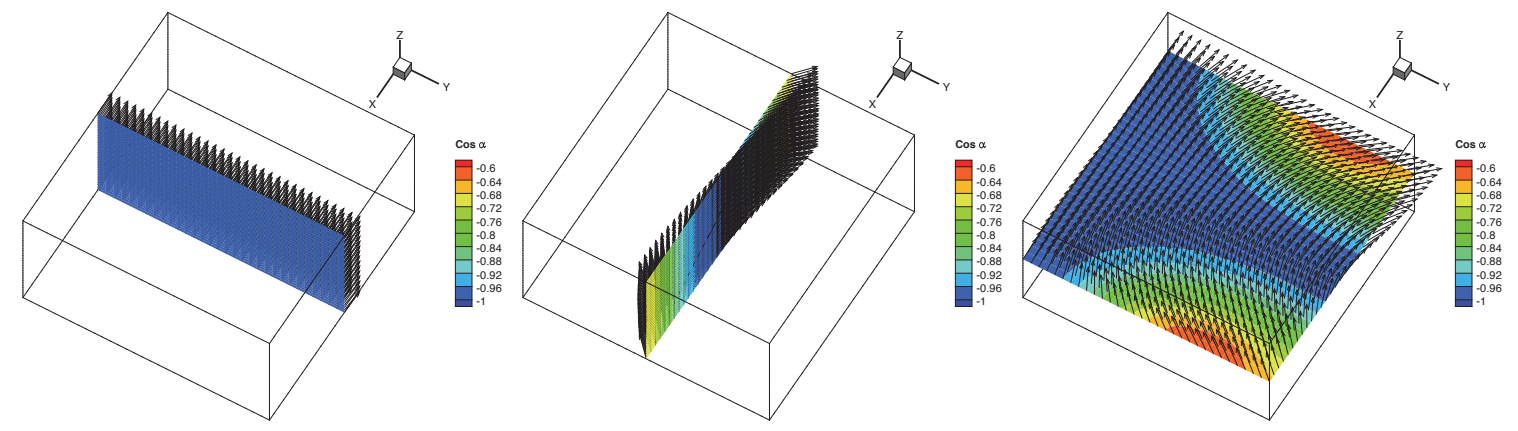

(c) Size $=21\left(192 \times 192 \times 96 \mathrm{~nm}^{3}\right)$
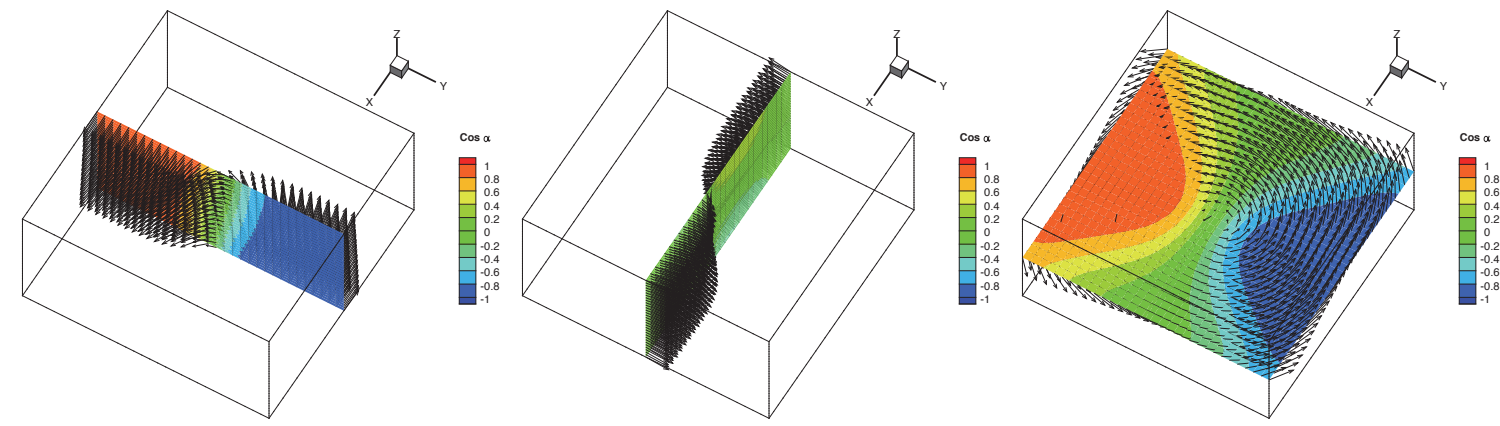

(d) Size $=51\left(467 \times 467 \times 236.5 \mathrm{~nm}^{3}\right)$

However, as $n$ is increased to 11 (Fig. 5b), the moments tend to show a pattern of deviation from $<100>$ directions. The type of pattern in Fig. 5b, has been described as a "flower" pattern in previous simulations [4,19]. Each moment in Fig. 5b represents a collection of $11 \times 11 \times 11$ unit cells of iron, and therefore the demagnetizing force is now significantly larger than in Fig. 5a. At $n=21$ (Fig. 5c) the deviation pattern becomes more significant in all three principal planes and the systematic rotation of the moments to change direction typically seen in a domain wall develop, although the particle size is not big enough to support a fully developed domain wall. Finally, at $n=51$ (Fig. $5 \mathrm{~d}$ ) a fully developed vortex pattern is seen with the formation of $180^{\circ}$ domain wall on planes normal to the $x$ - and $y$ directions. The domain pattern shown in Fig. $5 \mathrm{~d}$ is similar to those obtained in recent phase field simulations in iron that show four $90^{\circ}$ domains that come together roughly at the midpoint of the surface normal to the z-direction to form a 
vortex like pattern [12]. However, there is an important difference between the current result and the domain structure shown in ref. [12] where periodic boundary conditions were used in order to apply the FFT method for calculating the demagnetizing field. The periodic boundary conditions lead to the complete aligning of the moments on the boundaries of the simulation domain, while the non-periodic boundary conditions result in a systematic rotation of the boundary moments. A similar structure is also shown to exist using non-periodic boundary conditions and a finite element approach to solve the LLG equations [21]. Based on the results shown in Fig. 5 d, the width of the $180^{\circ}$ domain wall was measured to be approximately $160 \mathrm{~nm}$, which is in close agreement with experimentally measured value for Fe-3wt.\%Si using the adiabatic spin-flip effect on neutron diffraction [22]. $E_{d i p}$ and $E_{e x}$ are not expected to change significantly with $3 \mathrm{wt} . \%$ addition of Si to iron.
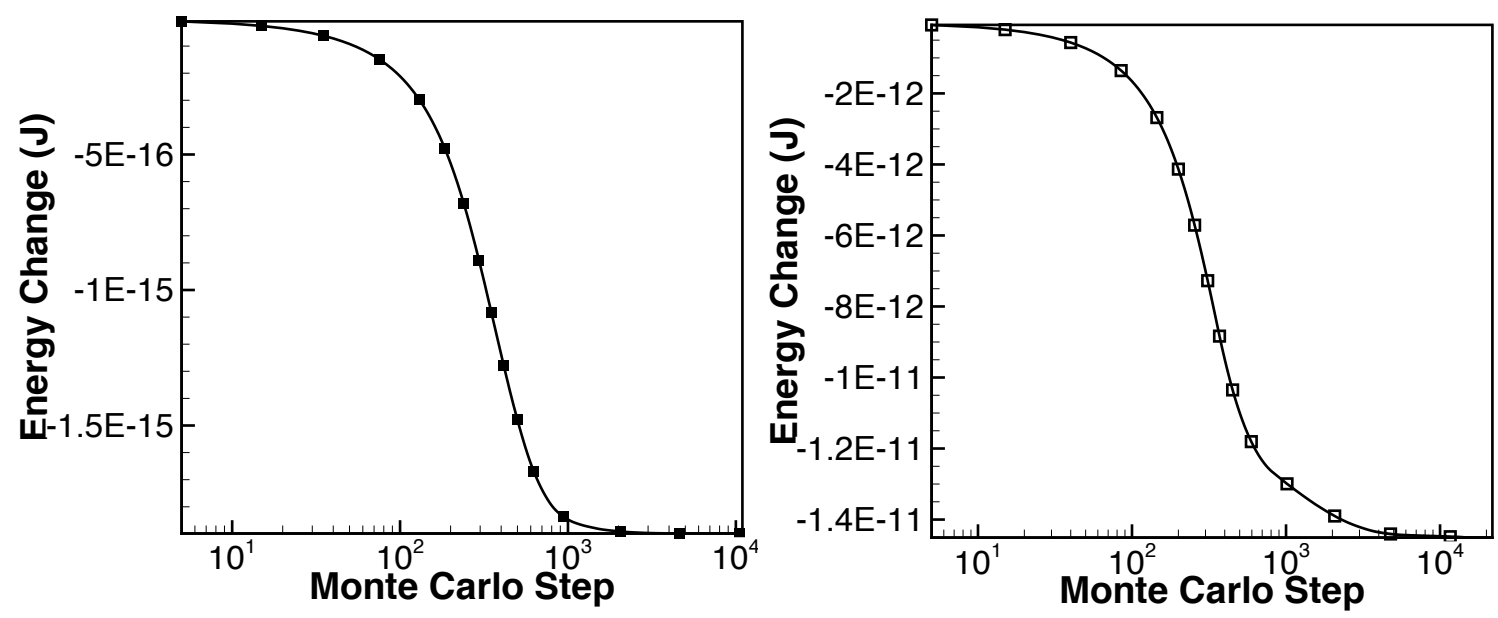

Figure 6. Change in the total system energy due to evolution of magnetic moments for $n=1$ (left) and $n=51$ (right).

The energy change in the system due to the evolution of the moments is shown in Fig. 6 . The aligning of moments along the $x$-direction for $n=1$ and the formation of the vortex structure for $n=51$ both result in the minimization of the total magnetic energy of the system. The temporal evolution of the total magnetic moments in the $x, y$ and $z$ directions is shown in Fig. 7 for $n=1$ and $n=51$. For $n=51$, the total moments in all three directions go to zero, as expected for a vortex structure. It is important to recognize the significance of the $2^{\text {nd }} \mathrm{MC}$ move that involves a random rotation of all the moments in the domain. If such a move is not included, the moments in the $n=1$ case (Fig. 6 a) could align along any random direction since $E_{d i p}$ is much larger than $E_{a n}$. However, the cluster rotation ensures further reduction in the energy due to the rotation of the moments towards an easy magnetization direction.

The accuracy of the proposed scaling law for $E_{\text {dip }}$ can be further improved by extending the atomistic calculation to larger simulation volume. However, even with 
the available HPC resources it becomes difficult to extend the size scale much further. In the future, we will employ the fast multipole method [13] in a parallel computing environment to significantly extend the simulation volume. The ultimate goal of this research is to extend the current approach to polycrystalline grain structures in soft magnetic materials with grain sizes in the micrometer length scale.

\section{Summary and Conclusions}

A new scaling approach is proposed for the contributions due to spin exchange energy, dipole-dipole interaction energy and the magneto-crystalline anisotropy energy as the size of the simulation domain increases. The scaling laws along with atomistic MC simulation under HPC environment is used to simulate the transition from a mono-domain to the flower state to the vortex state in single crystal iron that is in close agreement with previously simulated and experimentally measured values. The width of a $180^{\circ}$ domain wall from the vortex structure calculated from the simulated structure was roughly $160 \mathrm{~nm}$ in excellent agreement with experimentally measured value for $\mathrm{Fe}-3 \% \mathrm{Si}$. 


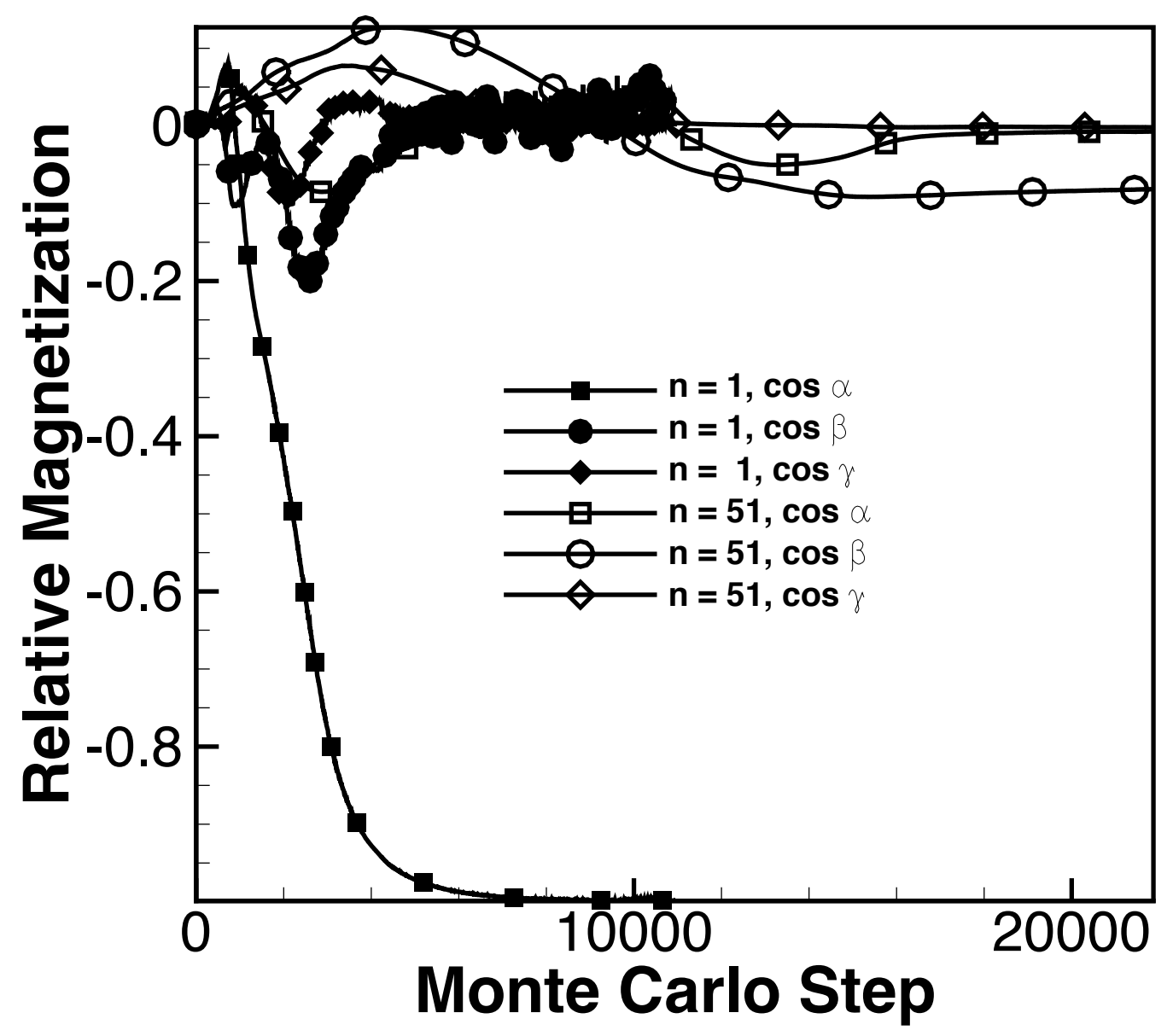

Figure 7. Temporal evolution of the magnetic moments in the $\mathrm{x}, \mathrm{y}$ and $\mathrm{z}$ directions for $n=1$ and $n=51$

Acknowledgements

This research was sponsored by Research sponsored by the U.S. Department of Energy, Office of Energy Efficiency and Renewable Energy, Vehicle Technologies Office, under contract DE-AC05-000R22725 with UT-Battelle, LLC. This research used resources of the Oak Ridge Leadership Computing Facility, which is a DOE Office of Science User Facility supported under Contract DE-AC05-000R22725.

\section{References}

1. S. Müller-Pfeiffer, M. Schneider and W. Zinn, Physical Review B, vol. 49, 1994, pp. 15745-15752.

2. H. Kronmüller and R. Hertel, Journal of Magnetism and Magnetic Materials, vol. 215-216, 2000, pp. 11-17.

3. M.E. Schabes and H.N. Bertram, Journal of Applied Physics, vol. 64, 1988, pp. 1347-1357. 
4. R. Hertel and H. Kronmüller, Journal of Magnetism and Magnetic Materials, vol. 238, 2002, pp. 185-199.

5. A. Kákay and L.K. Varga, Journal of Applied Physics, vol. 97, 2005, 083901.

6. K. yamaguchi, K. Yamada and T. Takagi, IEEE Transactions on Magnetics, vol. 38, 2002, pp. 865-868.

7. F. Matsubara, S. Endoh and T. Sasaki, Journal of the Physical Society of Japan, vol. 72, 2003, pp. 1326-1329.

8. K. Terashima, K. Suzuki and K. Yamaguchi, Physica B, vol. 486, 2016, pp. 52-56.

9. D. Hinzke and U. Nowak, Journal of Magnetism and Magnetic Materials, vol. 221, 2000, pp. 365-372.

10. T. Koyama, Science and Technology of Advanced Materials, vol. 9, 2008, 013006.

11. S. Hu, et al., IEEE Magnetics Letters, vol. 4, 2013, 3500104.

12. Y. Li et al., Journal of Physics D: Applied Physics, vol. 48, 2015, 305001.

13. P.B. Visscher and D.M. Apalkov, Journal of Magnetism and Magnetic Materials, vol. 322, 2010, pp. 275-281.

14. D. Pekurovsky, SIAM Journal of Scientific Computing, vol. 34, 2012, pp. C192C209.

15. P. Vargas, D. Altbir and J. d'Albuquerque e Castro, Physical Review B, vol. 73, 2006, 092417.

16. E.A. Velásquez, et al., Physical Review B, vol. 91, 2015, 134418.

17. N. Metropolis, et al., Journal of Chemical Physics, vol. 21, 1953, pp. 1087-1092.

18. J. Yin, M. Eisenbach, D.M. Nicholson and A. Rusanu, Phys. Rev. B., vol. 86, 2012, 214423.

19. Gatel, et al., Nano Letters, vol. 15, 2015, pp. 6952-6957.

20. Wachowiak, et al., Science, vol. 298, 2002, pp. 577-580.

21. J. Wang and J. Zhang, International Journal of Solids and Structures, vol. 50, 2013, pp. 3597-3609.

22. K.M. Podurets and S.S. Shilstein, Physica B, vol. 297, 2001, pp. 263-267. 